\title{
Antimicrobial Activity of Minocycline-Loaded Genipin-Crosslinked Nano-Fibrous Chitosan Mats for Guided Tissue Regeneration
}

\author{
Peter A. Norowski ${ }^{1}$, Jegdish Babu ${ }^{2}$, Pradeep C. Adatrow ${ }^{3}$, Franklin Garcia-Godoy ${ }^{2}$, \\ Warren O. Haggard ${ }^{1}$, Joel D. Bumgardner ${ }^{1}$
}

${ }^{1}$ Department of Biomedical Engineering, Herff College of Engineering, University of Memphis, Memphis, USA; ${ }^{2}$ Department of Bioscience Research, College of Dentistry, University of Tennessee Health Science Center, Memphis, USA; ${ }^{3}$ Department of Periodontology, College of Dentistry, University of Tennessee Health Science Center, Memphis, USA.

Email: jbmgrdnr@memphis.edu

Received August $8^{\text {th }}, 2012$; revised September $17^{\text {th }}, 2012$; accepted September $27^{\text {th }}, 2012$

\begin{abstract}
Antimicrobial delivery has been advocated for guided tissue regeneration (GTR) or guided bone regeneration (GBR) therapies involving patients with aggressive or unresolved periodontitis/peri-implantitis. Electrospun chitosan membranes demonstrate several advantages over traditional GTR barrier membranes because they stimulate healing, mimic the topology of the extracellular matrix, and allow for diffusion of nutrients and wastes into/out of the graft site, and were shown to stimulate bone formation in a rabbit calvarial critical-size defect model. Previously, we have shown improvements in mechanical properties and degradation kinetics by crosslinking electrospun membranes with $5 \mathrm{mM}$ or 10 $\mathrm{mM}$ genipin. We have also demonstrated the ability of elecrospun chitosan membranes to inhibit lippopolysaccharide (LPS)-induced monocyte activation. In this study, minocycline was incorporated into the chitosan membrane by passive absorption at 5 or $10 \mathrm{mg} / \mathrm{mL}$. The minocycline-loaded membranes and control membranes (carrier only) were tested against Porphyromonas gingivalis ( $P$. gingivalis) by repeated zone of inhibition (ZOI) measurements. Testing showed that uncrosslinked and genipin-crosslinked membranes have similar capacity to absorb aqueous solutions (swelling ratio 1.7 - 2.2). Minocycline loading resulted in bacterial inhibition for up to 8 days from crosslinked membranes (with 11 $\mathrm{mm}$ initial ZOI) whereas uncrosslinked membranes loaded with minocycline only inhibited bacteria for 4 days (with 8 $\mathrm{mm}$ initial ZOI). These in vitro results suggest that genipin-crosslinked electrospun chitosan membranes loaded with minocycline may be able to reduce early bacterial contamination of GTR graft sites.
\end{abstract}

Keywords: Chitosan; Nanofiber; Genipin; Minocycline; Guided Tissue Regeneration

\section{Introduction}

Bone graft materials are widely used to help repair bone defects in periodontitis. Guided bone regeneration (GBR) and guided tissue regeneration (GTR) barrier membranes are often used to help maintain space for newly forming bone and to prevent the infiltration of soft tissues into the bone graft space. However, a major problem with current GBR/GTR membranes is that they often become exposed and are susceptible to infection which can reduce amount of regenerated bone. Hence, there is a need for new method to prevent infection of GBR/GTR membranes and regenerating alveolar bone. Electrospun chitosan and other chitosan membrane materials have been advocated for GBR/GTR barrier membranes [1-3]. Chitosan is advantageous because it has been reported to stimulate the healing of dental pulp wounds, [4] is osteogenic, and has been shown to inhibit lippopolysaccharide (LPS)-induced monocyte activation $[5,6]$. Electrospun material are advantageous because they mimic the topology of the extracellular matrix, [7] allow the diffusion of nutrients and waste, have a small enough pore size to limit cellular infiltration, and also have the capability to regenerate dense cortical bone in animal models [8].

We have developed electrospun chitosan membranes with genipin cross-linking (natural crosslinker) that have resulted in $12 \%$ reduction in mass loss over 16 weeks, and increased the tensile strength of the membranes three fold [9]. We have also demonstrated that the genipin crosslinked chitosan membranes were not cytotoxic to osteoblast or fibroblast cells, and did not cause monocyte activation. We have also shown the ability of genipin 
Nano-Fibrous Chitosan Mats for Guided Tissue Regeneration

crosslinked electropun chitosan membranes to inhibit the LPS-induced release of nitric oxide (NO) from RAW 264.7 monocyte cells over a 3 day period [10]. NO expression is elevated in the periodontal and gingival tissues of patients with periodontitis and its inhibition is a potential therapeutic target $[11,12]$.

Because of the hydrophilic nature of chitosan and the high surface area of electrospun fibers, electrsopun chitosan membranes may also serve as an effective antibiotic delivery vehicle. The ability of the membranes to prevent infection through the local delivery of antibiotics during bone healing of the site while also preventing soft tissue penetration may lead to a significant improvement in clinical bone healing in patients. Minocycline is commonly used in periodontal therapy as an antimicrobial agent but it also has the ability to limit tissue destruction, by inhibition of tissue destroying enzymes such as collagenase, MMP-2 and MMP-9 [13].

In this study, uncrosslinked and genipin crosslinked electrospun chitosan membranes were impregnated by immersion in $10 \mathrm{mg} / \mathrm{mL}$ minocycline or $5 \mathrm{mg} / \mathrm{mL}$ minocycline and tested against Porphyromonas gingivalis $(P$. gingivalis) by zone of inhibition. $P$. gingivalis is an important and extensively studied periodontal pathogen involved in the pathogenesis of periodontitis. In addition, swelling was measured to assess the capacity of the electrospun membranes to absorb aqueous solutions.

\section{Materials and Methods}

\subsection{Electrospinning Procedure}

Electrospun chitosan nanofibrous mats were fabricated as previously described (Norowski et al., in review). Briefly, a $5.50 \mathrm{wt} \%$ chitosan solution in $70(\mathrm{v} / \mathrm{v}) \%$ trifluoroacetic acid and $30(\mathrm{v} / \mathrm{v}) \%$ methylene chloride was mixed with genipin for 30 minutes prior to the start of electrospinning. The genipin concentrations investigated were 0,5 , or $10 \mathrm{mM}$. The solution was electrospun at $25 \mathrm{kV}$ and the fibers were collected on a non-stick aluminum foil target (Reynolds wrap ${ }^{\circledR}$ ) rotated at 8.4 RPM by an AC motor to ensure even and random distribution of fibers. After electrospinning, the nano-fibrous mat was put under vacuum overnight to remove residual solvent, removed from the foil, and then neutralized at room temperature in $5 \mathrm{M} \mathrm{Na}_{2} \mathrm{CO}_{3}$ (saturated solution) for 3 hours [14]. Membranes were sterilized by ethylene oxide gas.

\subsection{Characterization of Nano-Fibrous Membrane}

\subsubsection{Swelling}

The swelling index of the nanofibrous membranes was determined by a swelling test. Swelling in phosphate buffered saline (PBS) was evaluated to estimate the amount of antibiotic solution that could be absorbed by the electrospun membranes. To determine the dry weight, membranes were maintained at $40^{\circ} \mathrm{C}$ overnight in a drying oven. After measuring the dry weight, membranes were submerged in PBS for $1 \mathrm{hr}$ ( \pm 15 minutes) to ensure complete swelling $(\mathrm{n}=4-5)$. Swelling index was calculated by $\left(\mathrm{Wt}_{\mathrm{WET}}-\mathrm{Wt}_{\mathrm{DRY}}\right) / \mathrm{Wt}_{\mathrm{DRY}}$.

\subsubsection{Minocycline Loading}

Minocycline was loaded into chitosan nano-fibrous membranes by passive absorption. Pre-cut, pre-sterilized circular specimens (10 $\mathrm{mm}$ diameter), were submersed in minocycline solution (10 or $5 \mathrm{mg} / \mathrm{mL}$ in de-ionized water) for 15 minutes. Negative controls were submersed in deionized water only. Minocycline solutions were weighed before and after membrane swelling to determine the amount of antibiotic solution absorbed.

\subsubsection{P. gingivalis Zone of Inhibition (ZOI)}

The model periodontal pathogen used in this study was Porphyromonas gingivalis (ATCC No. 33277) which was originally isolated from human gingival sulcus. Bacteria were maintained as frozen stock cultures and grown anaerobically at $37^{\circ} \mathrm{C}$ in trypticase soy broth (BD BBL, Franklin Lakes, NJ, USA) supplemented with $1 \mathrm{~g}$ of yeast extract per liter, $5 \mathrm{mg}$ of hemin per liter, and $1 \mathrm{mg}$ of menadione per liter. After 72 hours of growth, bacteria were collected and resuspended to contain $1 \times 10^{7}$ cells $/ \mathrm{ml}$. A suspension $(0.5 \mathrm{ml})$ of this stock suspension was spread on a blood agar plate (BD BBL, Franklin Lakes, NJ, USA) and the electrospun chitosan mats loaded with minocycline were placed onto the agar and incubated in an anaerobic jar with an anaerobic pack. Plates were checked development of a ZOI by sequentially placing membranes on freshly seeded bacterial lawns and recording ZOI at days 1, 4, 6, 8 and $11(\mathrm{n}=5)$. Zones were measured in mm. For swelling and ZOI tests, statistical differences were detected by ANOVA with Tukey's test used for post-hoc analysis $(\alpha=0.05)$.

\section{Results}

\subsection{Swelling}

Swelling experiments demonstrated that the electrospun membranes have a swelling ratio $2.24 \pm 0.57,1.71 \pm 0.47$, and $1.85 \pm 0.41$ for uncrosslinked, $5 \mathrm{mM}$ crosslinked and $10 \mathrm{mM}$ genipin-crosslinked membranes, respectively. The amount of swelling that occurred was not significantly affected by crosslinking $(p=0.29)$. Although swelling was allowed to occur for 1 hour to ensure complete swelling, the membranes appeared to be fully hydrated within 5 minutes (data not shown). 


\subsection{Minocycline Loading}

Submersion in $10 \mathrm{mg} / \mathrm{mL}$ minocycline solution resulted in a range of $0.12-0.52,0.53-0.54$, and $0.38-0.57 \mathrm{mg}$ of minocycline uptake for uncrosslinked, $5 \mathrm{mM}$ crosslinked and $10 \mathrm{mM}$ genipin-crosslinked membranes, respectively.

\section{3. $P$. gingivalis Zone of Inhibition (ZOI)}

ZOI testing demonstrated extended release of mino- cycline from the barrier membrane in vitro for up to 8 days after soaking in $10 \mathrm{mg} / \mathrm{mL}$ (Figure 1) or $5 \mathrm{mg} / \mathrm{mL}$ (Figure 2) minocycline for 15 minutes. It was noted that uncrosslinked membranes only remained bacteriostatic for 4 days as compared to the $5 \mathrm{mM}$ and $10 \mathrm{mM}$ crosslinked membranes which remained bacteriostatic for 8 days. The ZOI from $5 \mathrm{mM}$ and $10 \mathrm{mM}$ crosslinking were similar although $10 \mathrm{mM}$ crosslinking did result in a larger ZOI than $5 \mathrm{mM}$ on day 4 when loaded with 10 $\mathrm{mg} / \mathrm{mL}$ minocycline. None of the negative controls (car-

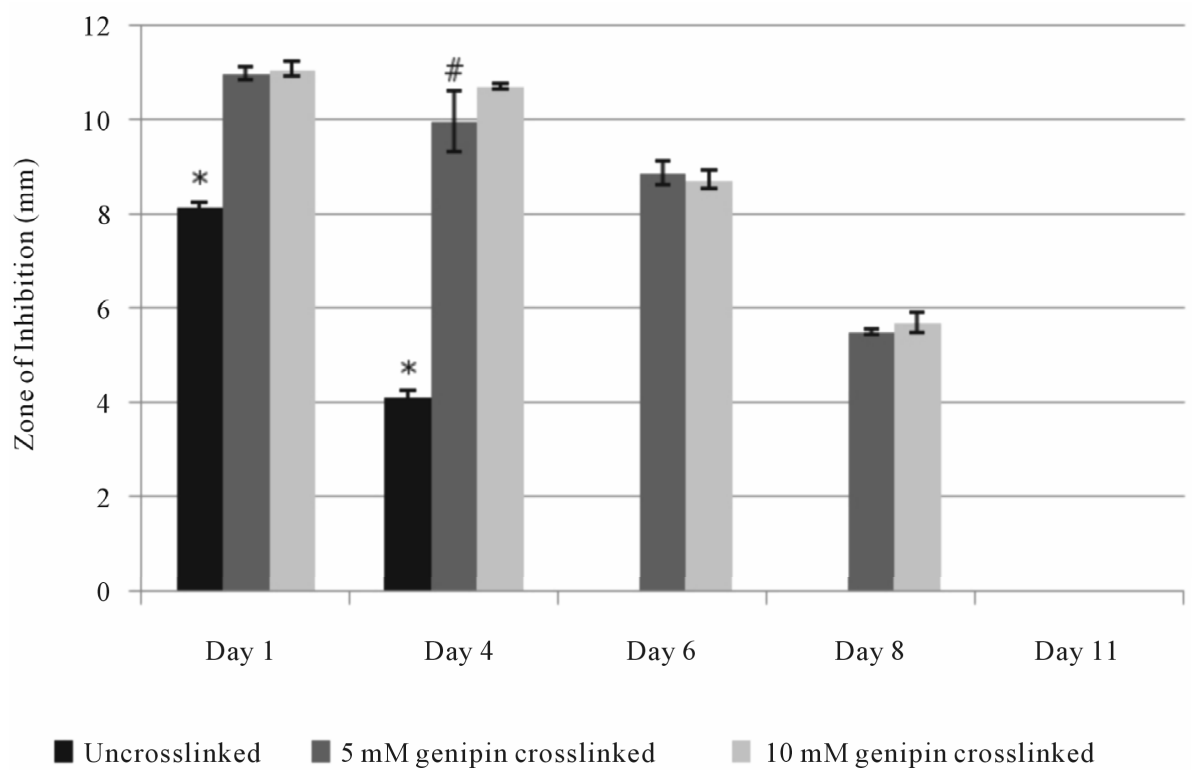

Figure 1. Inhibition of $P$. gingivalis over an 11 day period by electrospun chitosan membrane loaded with $10 \mathrm{mg} / \mathrm{mL}$ minocycline. Error bars represent the standard deviation $(n=5)$ * and \# denote statistical differences $(\alpha=0.05)$.

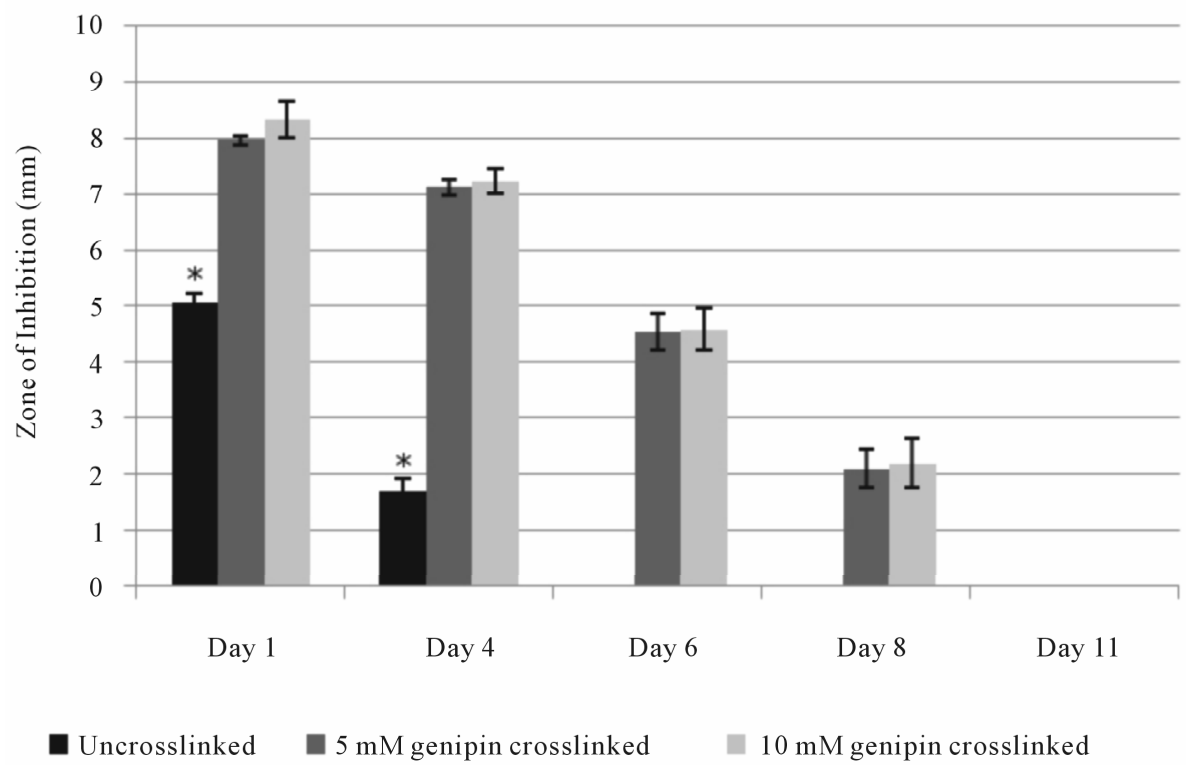

Figure 2. Inhibition of $P$. gingivalis over an 11 day period by electrospun chitosan membrane loaded with 5 mg/mL minocycline. Error bars represent the standard deviation $(n=5)$. *denotes statistical difference $(\alpha=0.05)$. 
rier only) produced zones of inhibition.

\section{Discussion}

The swelling study demonstrated that membranes have similar capacity to absorb fluid and any differences in swelling capacity were not significant. Therefore, the crosslinked membrane should have similar capacity to absorb drugs, antibiotics, or growth factor solutions. Unlike vapor crosslinking and solution crosslinking methods, where materials are exposed to a crosslinking agent that crosslinks polymer chains primarily on the surface, in this study, the crosslinking agent is dispersed within the polymer solution used for scaffold fabrication. This method creates a more uniformly and thoroughly crosslinked membrane, which likely contributed to improved antibiotic retention as observed in this study and more uniform degradation kinetics. A previous examination using x-ray diffraction showed that crystallinity was decreased during crosslinking. This may have contributed to slightly lower swelling volumes, however this difference was not significant.

In this study, genipin-crosslinked electrospun chitosan was able to absorb minocycline and release it in an extended manner that remained bacteriostatic for longer periods than uncrosslinked membranes ( 8 days as compared to 4 days). These results are similar to reports by others who have loaded biodegradable GTR membranes with antibiotics/antiseptics such as tetracycline, doxycycline or chlorhexidine [15-17]. Delayed degradation kinetics (Norowski et al., in review) contributed to the extended release seen from crosslinked membranes. Thus, uncrosslinked chitosan membranes degraded faster and resulted in lower minocycline loading and release levels.

One clinical investigation reported no improvements in clinical parameters associated with the local application of minocycline ointment before GTR therapy, but this study did not investigate minocycline incorporation into the GTR membrane itself, and only investigated the use of type 1 collagen membranes. [18] Other investigations with a non-membrane local delivery system demonstrated improvement in clinical parameters associated with the use of minocycline microcapsules (Arestin ${ }^{\circledR}$ ). [19] This microcapsule study also showed that reduction in periodontal pocket probing depth (improved clinical outcomes) correlated strongly with the ability to inhibit red complex bacteria in vitro, a sub-group of periodontal pathogens that includes $P$. gingivalis, $T$. forsythia, and $T$. denticola [19].

\section{Conclusion}

In this study, we have shown the ability of genipin-crosslinked electrospun chitosan to deliver clinically relevant levels of minocycline over an 8 day period. The eluted minocycline was able to inhibit growth of $P$. gingivalis, a model periopathogen, in vitro. Crosslinked membranes released inhibitory levels of minocycline for 8 days while, uncrosslinked membranes only inhibited growth for 4 days. This prolonged minocycline elution profile suggests that genipin-crosslinking improved the drug-carrier properties of electrospun chitosan.

\section{REFERENCES}

[1] S. M. Kuo, S. J. Chang, T. W. Chen and T. C. Kuan, "Guided Tissue Regeneration for Using a Chitosan Membrane: An Experimental Study in Rats," Journal of Biomedical Materials Research Part A, Vol. 76, No. 2, 2006, pp. 408-415. doi:10.1002/jbm.a.30534

[2] S. Y. Shin, H. N. Park, K. H. Kim, M. H. Lee, Y. S. Choi, Y. J. Park, Y. M. Lee, Y. Ku, I. C. Rhyu, S. B. Han, S. J. Lee and C. P. Chung, "Biological Evaluation of Chitosan Nanofiber Membrane for Guided Bone Regeneration," Journal of Periodontology, Vol. 76, No. 10, 2005, pp. 17781784. doi:10.1902/jop.2005.76.10.1778

[3] Y. J. Yeo, D. W. Jeon, C. S. Kim, S. H. Choi, K. S. Cho, Y. K. Lee and C. K. Kim, "Effects of Chitosan Nonwoven Membrane on Periodontal Healing of Surgically Created One-Wall Intrabony Defects in Beagle Dogs," Journal of Biomedical Materials Research Part B: Applied Biomaterials, Vol. 72, No. 1, 2005, pp. 86-93. doi:10.1002/jbm.b.30121

[4] T. Matsunaga, K. Yanagiguchi, S. Yamada, N. Ohara, T. Ikeda and Y. Hayashi, "Chitosan Monomer Promotes Tissue Regeneration on Dental Pulp Wounds," Journal of Biomedical Materials Research Part A, Vol. 76, No. 4, 2005, pp. 711-720.

[5] H. J. Yoon, M. E. Moon, H. S. Park, S. Y. Im and Y. H. Kim, "Chitosan Oligosaccharide (COS) Inhibits LPS-Induced Inflammatory Effects in RAW 264.7 Macrophage Cells," Biochemical and Biophysical Research Communications, Vol. 358, No. 3, 2007, pp. 954-959. doi:10.1016/j.bbrc.2007.05.042

[6] K.-S. Nam, M.-K. Kim and Y.-H. Shon, "Inhibition of Proinflammatory Cytokine-Induced Invasiveness of HT29 Cells by Chitosan Oligosaccharide," Journal of Microbiology and Biotechnology, Vol. 17, No. 12, 2007, pp. 2042-2045.

[7] F. Chen, X. Li, X. Mo, C. He, H. Wang and Y. Ikada, "Electrospun Chitosan-P(LLA-CL) Nanofibers for Biomimetic Extracellular Matrix," Journal of Biomaterials Science, Vol. 19, No. 5, 2008, pp. 677-691. doi:10.1163/156856208784089661

[8] Y. Z. Cai, L. L. Wang, H. X. Cai, Y. Y. Qi, X. H. Zou and H. W. Ouyang, "Electrospun Nanofibrous Matrix Improves the Regeneration of Dense Cortical Bone," Journal of Biomedical Materials Research Part A, Vol. 95, No. 1, 2010, pp. 49-57. doi:10.1002/jbm.a.32816

[9] P. A. Norowski, T. Fujiwara, W. C. Clem, P. C. Adatrow, E. C. Eckstein, W. O. Haggard and J. D. Bumgardner, 
"Novel Naturally Crosslinked Electrospun Nanofibrous Chitosan Mats for Guided Bone Regeneration Membranes: Material Characterization and Cytocompatibility," Journal of Tissue Engineering and Regenerative Medicine, E-Ahead of Print, 2012.

[10] P. A. Norowski, S. Mishra, P. C. Adatrow, W. O. Haggard and J. D. Bumgardner, "Suture Pullout Strength and in Vitro Fibroblast and RAW 264.7 Monocyte Biocompatibility of Genipin Crosslinked Nanofibrous Chitosan Mats for Guided Tissue Regeneration," Journal of Biomedical Materials Research Part A, Vol. 100A, No. 11, 2012, pp. 2890-2896. doi:10.1002/jbm.a.34224

[11] D. Uğar-Çankal and N. Ozmeric, "A Multifaceted Molecule, Nitric Oxide in Oral and Periodontal Diseases," Clinica Chimica Acta, Vol. 366, No. 1-2, 2006, pp. 90100. doi:10.1016/j.cca.2005.10.018

[12] F. Daghigh, R. C. Borghaei, R. D. Thornton and J. H. Bee, "Human Gingival Fibroblasts Produce Nitric Oxide in Response to Proinflammatory Cytokines," Journal of Periodontology, Vol. 73, No. 4, 2002, pp. 392-400. doi:10.1902/jop.2002.73.4.392

[13] B. R. Rifkin, A. T. Vernillo and L. M. Golub, "Blocking Periodontal Disease Progression By Inhibiting TissueDestructive Enzymes: A Potential Therapeutic Role for Tetracyclines and Their Chemically-Modified Analogs," Journal of Periodontology, Vol. 64, No. 8, 1993, pp. 819-827. doi:10.1902/jop.1993.64.8s.819

[14] P. Sangsanoh and P. Supaphol, "Stability Improvement of Electrospun Chitosan Nanofibrous Membranes in Neutral or Weak Basic Aqueous Solutions," Biomacromolecules,
Vol. 7, No. 10, 2006, pp. 2710-2714. doi:10.1021/bm0602861

[15] C.-Y. Chang and S. Yamada, "Evaluation of the Regenerative Effect of a 25\% Doxycycline-Loaded Biodegradable Membrane for Guided Tissue Regeneration," Journal of Periodontology, Vol. 71, No. 7, 2000, pp. 10861093. doi:10.1902/jop.2000.71.7.1086

[16] Y.-T. Chen, S.-L. Hung, L.-W. Lin, L.-Y. Chi and L.-J. Ling, "Attachment of Periodontal Ligament Cells to Chlorhexidine-Loaded Guided Tissue Regeneration Membranes," Journal of Periodontology, Vol. 74, No. 11, 2003, pp. 1652-1659. doi:10.1902/jop.2003.74.11.1652

[17] Y. J. Park, Y. M. Lee, S. N. Park, J. Y. Lee, Y. Ku, C. P. Chung and S. J. Lee, "Enhanced Guided Bone Regeneration by Controlled Tetracycline Release from Poly(LLactide) Barrier Membranes," Journal of Biomedical Materials Research, Vol. 51, No. 3, 2000, pp. 391-739. doi:10.1002/1097-4636(20000905)51:3<391::AID-JBM1 3>3.0.CO;2-9

[18] M. Minabe, T. Kodama, T. Kogou, H. Fushimi, T. Sugiyama, K. Takeuchi, E. Miterai and S. Nishikubo, "Clinical Significance of Antibiotic Therapy in Guided Tissue Regeneration with a Resorbable Membrane," Periodontal Clinical Investigations, Vol. 23, No. 1, 2001, pp. 20-30.

[19] P. S. Bland, J. M. Goodson, J. C. Gunsolley, S. G. Grossi, J. Otomo-Corgel, F. Doherty and J. L. Comiskey, "Association of Antimicrobial and Clinical Efficacy: Periodontitis Therapy with Minocycline Microspheres," Journal of the International Academy of Periodontology, Vol. 12, No. 1, 2010, pp. 11-19. 